\title{
Role of Aquaporin 3 in hydrogen peroxide transport and cellular signaling
}

\section{Mariko Hara-Chikuma}

\section{Department of Pharmacology, Keio University School of Medicine, Japan}

Aquaporin-3 (AQP3) is expressed in various cell types, such as epithelial cells, immune cells and cancer cells. AQP3 has the primary function of transporting water and small molecules including glycerol and hydrogen peroxide (H2O2). We have shown the involvement of AQP3 expression in the development of inflammatory disease including psoriasis, asthma, or liver injury, and cancer progression via $\mathrm{H} 2 \mathrm{O} 2$ transporting function. We demonstrated that AQP3-mediated cellular $\mathrm{H} 2 \mathrm{O} 2$ exert as a second messenger for $\mathrm{H} 2 \mathrm{O} 2$ regulated cell signaling such as NF-kB or PTEN, which contributed to inflammation or cell growth.

Recently, we established monoclonal mouse AQP3 inhibitory antibody (mAb-AQP3), which suppressed cell growth and $\mathrm{H} 2 \mathrm{O} 2$-dependent cell signaling. We also found that mAb-AQP3 can prevent the acute and chronic liver injury, and cancer cell growth in mice model.

In this session, we will show that the AQP3 inhibition is a novel therapeutic target in inflammatory disease such as liver injury or cancer progression, and discuss the underlying mechanism of AQP3 inhibition. 